\title{
Approaching the Dirac point in high mobility multilayer epitaxial graphene
}

\author{
M. Orlita,,${ }^{1,2,3, *}$ C. Faugeras, ${ }^{1}$ P. Plochocka,${ }^{1}$ P. Neugebauer,${ }^{1}$ G. Martinez,${ }^{1}$ D. K. \\ Maude, ${ }^{1}$ A.-L. Barra,${ }^{1}$ M. Sprinkle, ${ }^{4}$ C. Berger,${ }^{4,5}$ W. A. de Heer ${ }^{4}$ and M. Potemski ${ }^{1}$ \\ ${ }^{1}$ Grenoble High Magnetic Field Laboratory, CNRS, BP 166, F-38042 Grenoble Cedex 09, France \\ ${ }^{2}$ Institute of Physics, Charles University, Ke Karlovu 5, CZ-121 16 Praha 2, Czech Republic \\ ${ }^{3}$ Institute of Physics, v.v.i., ASCR, Cukrovarnická 10, CZ-162 53 Praha 6, Czech Republic \\ ${ }^{4}$ School of Physics, Georgia Institute of Technology, Atlanta, Georgia 30332, USA \\ ${ }^{5}$ Institut Néel/CNRS-UJF BP 166, F-38042 Grenoble Cedex 9, France
}

(Dated: October 23, 2018)

\begin{abstract}
Multi-layer epitaxial graphene (MEG) is investigated using far infrared (FIR) transmission experiments in the different limits of low magnetic fields and high temperatures. The cyclotron-resonance like absorption is observed at low temperature in magnetic fields below $50 \mathrm{mT}$, allowing thus to probe the nearest vicinity of the Dirac point and to estimate the conductivity in nearly undoped graphene. The carrier mobility is found to exceed $250,000 \mathrm{~cm}^{2} /$ (V.s). In the limit of high temperatures, the well-defined Landau level (LL) quantization is observed up to room temperature at magnetic fields below $1 \mathrm{~T}$, a phenomenon unique in solid state systems. A negligible increase in the width of the cyclotron resonance lines with increasing temperature indicates that no important scattering mechanism is thermally activated, supporting recent expectations of high room-temperature mobilities in graphene.
\end{abstract}

The quality of electronic systems, measured in terms of the carrier mobility, and determined by carrier scattering processes, is a major issue in material science both from a fundamental physics and an applications point of view. This issue is now critical for further progress in the research on 2D allotropes of carbon [1, 2, 3, 4, 5, 6]. In exfoliated graphene, charged impurities are recognized as the dominant source of scattering in transport experiments $[7,[8,9]$. The presence of charged impurities even prevents experiments in the vicinity of the Dirac point, due to the electron and hole puddles which inevitably emerge in these samples when lowering density [10]. Recently, the first experiments on suspended graphene [11, 12], showed a promising way to come closer to Dirac point, but nevertheless, the exact role of individual scattering mechanisms, especially close to Dirac point, together with the nature of the minimum conductivity remains unclear [13, 14, 15, 16, 17].

In a parallel track to the exfoliated-graphene research, the equivalent problems arise in the study of epitaxial graphene. Multi-layer epitaxial graphene (MEG) can be relatively easily thermally decomposed from $\mathrm{SiC}$ on the C-terminated surface and consist of electrically decoupled graphene layers [18, 19]. It is highly conducting in the very close vicinity of the interface with $\mathrm{SiC}$, as a result of charge transfer from $\mathrm{SiC}$ 4, 20]. The subsequent layers are practically neutral 21]. Electrical measurements on epitaxial graphene samples probe mostly the low resistance interface layers. However, the quasi-neutral part of the MEG can be investigated by FIR optical absorption technique in an applied magnetic field [21].

Both epitaxial and exfoliated graphene have been extensively investigated at elevated temperatures. By analogy with room temperature ballistic transport in carbon nanotubes [22], very high mobilities are expected for graphene at room temperature. This is indeed suggested by the investigation of the temperature dependence of the extrinsic and intrinsic scattering mechanisms in exfoliated graphene 23] and in the weak temperature dependence of the resistance in epitaxial graphene [4].

In this Letter, we focus on carrier scattering processes in the majority graphene layers with a low carrier concentration and show their extraordinary quality, expressed by the temperature independent carrier mobility in excess of $250,000 \mathrm{~cm}^{2} /(\mathrm{V} . \mathrm{s})$. This information is extracted from the analysis of inter-LL transitions which are investigated in the relevant limits of high temperatures and low magnetic fields.

The investigated graphene sample was prepared by thermal decomposition from a $4 \mathrm{H}-\mathrm{SiC}$ substrate [1] and contains around $\sim 100$ graphene layers. The sample was characterized using micro-Raman experiment, which revealed similarly to Ref. 24] the presence of decoupled graphene layers as well as of additional graphite residuals. The Raman experiment with the laser spot of diameter $\sim 3 \mu \mathrm{m}$ shows an extraordinarily narrow $2 \mathrm{D}$ band $\left(\sim 20 \mathrm{~cm}^{-1}\right)$ compared to values reported on exfoliated graphene [25]. To measure the FIR transmittance of the sample, the macroscopic area of the sample $\left(\sim 4 \mathrm{~mm}^{2}\right)$ was exposed to the radiation of a globar or mercury lamp, which was analyzed by a Fourier transform spectrometer and delivered to the sample via light-pipe optics. In experiments performed at $T=2.0 \mathrm{~K}$, the light was detected by a composite bolometer placed directly below the sample, while at higher temperatures an external bolometer was used. All presented transmission spectra are normalized by the sample transmission at $B=0$.

The low temperature FIR transmission spectra of the investigated sample, shown in Fig. 1 are typical of the optical response of MEG [21]. Considering the LL spec- 


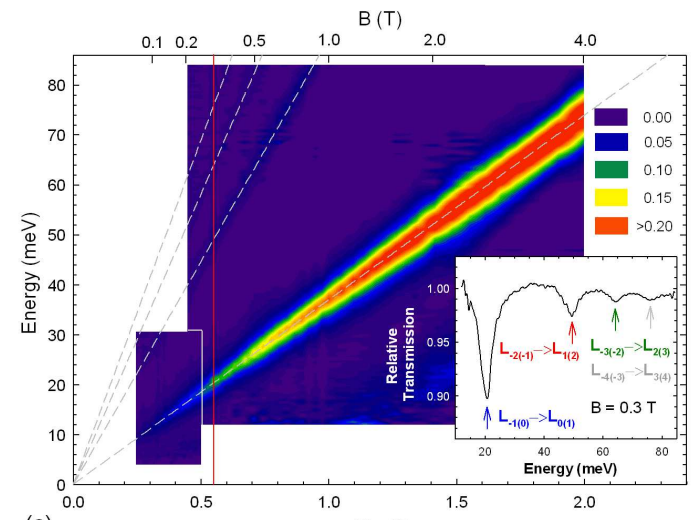$$
\text { (a) }
$$$$
B^{1 / 2}\left(T^{1 / 2}\right)
$$
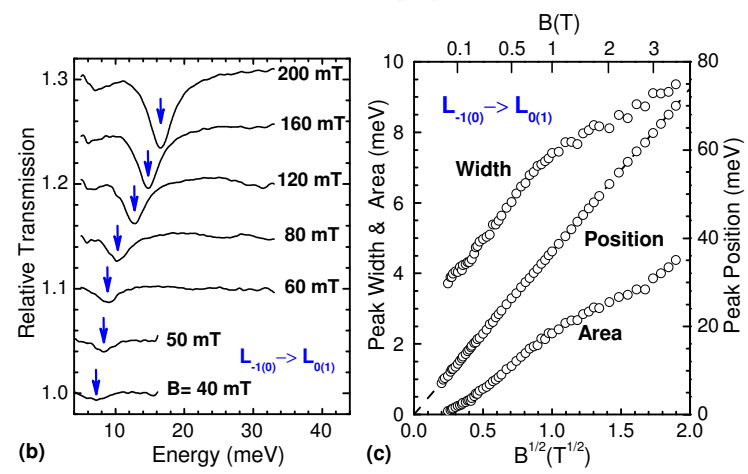

FIG. 1: (color online) Part (a): The FIR transmission $\mathcal{T}$ plotted as $-\ln \mathcal{T}$ as a function of the magnetic field at $T=2.0 \mathrm{~K}$. The dashed lines denote the expected transitions for $\tilde{c}=1.02 \times 10^{6} \mathrm{~m} \cdot \mathrm{s}^{-1}$. The inset shows the transmission spectrum at $B=0.3$ T. Part (b): FIR transmission taken at $T=2 \mathrm{~K}$ in low magnetic fields. For clarity, successive spectra are shifted vertically by 0.05 . The part (c) shows the peak position, width and area for the $\mathrm{L}_{-1(0)} \rightarrow \mathrm{L}_{0(1)}$ transition. The dashed line in part (c) is a least squares fit to the peak positions.

trum in graphene: $E_{n}=\operatorname{sign}(n) \tilde{c} \sqrt{2 e \hbar B|n|}$, four absorption lines, denoted by arrows in the inset of Fig.1(a) and showing a $\sqrt{B}$-scaled blueshift, can be identified as interLL transitions $\mathrm{L}_{-m} \rightarrow \mathrm{L}_{m+1}$ and $\mathrm{L}_{-(m+1)} \rightarrow \mathrm{L}_{m}$ with $m=0,1,2$ and 3 . The corresponding Fermi velocity was evaluated as $\tilde{c}=(1.02 \pm 0.01) \times 10^{6} \mathrm{~m} . \mathrm{s}^{-1}$. No deviations from the single particle model due to many-body effects are found in MEG in contrast to recent experiments performed on exfoliated samples [26, 27]. Notably, the spectra of this $\sim 100$ layer sample present no transitions symmetric around Dirac point $\left(\mathrm{L}_{-m} \rightarrow \mathrm{L}_{m}\right)$ which are characteristic of bulk graphite [28].

Henceforward, we focus on the main line in the spectra, $\mathrm{L}_{0(-1)} \rightarrow \mathrm{L}_{1(0)}$, which necessarily corresponds to transitions from or to the vicinity of the Fermi level. Following this transition with the magnetic field, we find no deviation from the $\sqrt{B}$-scaling and the line is still visible at $B \sim 40 \mathrm{mT}$ when it is centered at an energy of $\approx 7 \mathrm{meV}$. The FIR experiment, thus allows to probe the very close vicinity of Dirac point, hardly accessible in the current transport experiments [4, 11, 12], and also shows that the linearity of the density of states is preserved down to the distances of few meV from Dirac point. The disappearance of the line below $B \sim 40 \mathrm{mT}$ gives an estimation of the LL filling factor $\nu \approx 6$ at this field with the corresponding carrier density of $n_{0} \approx 5 \times 10^{9} \mathrm{~cm}^{-2}$. The possible remanent field of the solenoid of $\leq 5 \mathrm{mT}$ limits the accuracy of this estimation to $10 \%$. Note that this density is about three orders of magnitude smaller than the carrier density measured in transport for equivalent samples [1, 4, 29], but electrical conductance is governed by highly doped graphene layer(s) close to the SiC substrate. While the FIR experiment probes all layers in the sample, the strongly doped layer(s) give no contribution to the FIR spectra in the presented region of energies and magnetic fields.

The shape of the main line can be well reproduced by a simple Lorentzian curve. The results of the fitting procedure are shown in Fig. 1(c), where the peak position, area and width are plotted as a function of $\sqrt{B}$. Both the peak position and the area show a linear increase with $\sqrt{B}$, in agreement with expectations for a single graphene layer [21]. The latter also suggests a relatively good homogeneity of the carrier density, as the significant presence of more doped regions would result in a superlinear rise. An interesting evolution with magnetic field is seen for the linewidth $\delta E$. Starting from $\delta E \sim 4 \mathrm{meV}$ at the lowest magnetic field $B=60 \mathrm{mT}$, where the line-shape analysis is possible, the width increases nearly linearly with $\sqrt{B}$ up to almost $8 \mathrm{meV}$ at $1 \mathrm{~T}$. At higher magnetic fields, the increase continues but as a sublinear function of $\sqrt{B}$. The observed broadening cannot result from the electron-hole asymmetry, where a broadening which varies linearly with $B$ is expected [30]. The $\sqrt{B}$-dependent broadening of LLs was suggested by Shon and Ando [13] for the case of both short- and longrange scatterers, whose strength is independent of carrier density. Whereas the short-range scatterers should induce an identical broadening of all LLs, the $n=0 \mathrm{LL}$ should be broadened by a factor of $\sqrt{2}$ more than other LLs for long-range scatterers. As the width of the main line is not enhanced compared to the other transitions, we can conclude that short-range scattering is probably dominant here.

To compare our results with the recent transport experiments, a simple estimation of the scattering time, mobility and conductivity are presented. From the width of the main transition $\delta E$, the carrier scattering time can be estimated using $\tau=2 \hbar / \delta E$, also used to compare FIR transmission and transport experiments performed on exfoliated graphene 26, 31]. The scattering time, obtained for our macroscopic sample, is $\tau \sim 300$ fs at low magnetic fields $(\delta E \approx 4 \mathrm{meV})$ and decreases with increasing $B$ to $\tau \sim 150$ fs at $B=1 \mathrm{~T}(\delta E \approx 8 \mathrm{meV})$. Equivalent scattering times $\tau \sim 200$ fs are nowadays reported for single-flake graphene on a $\mathrm{Si} / \mathrm{SiO}_{2}$ substrate at densities 


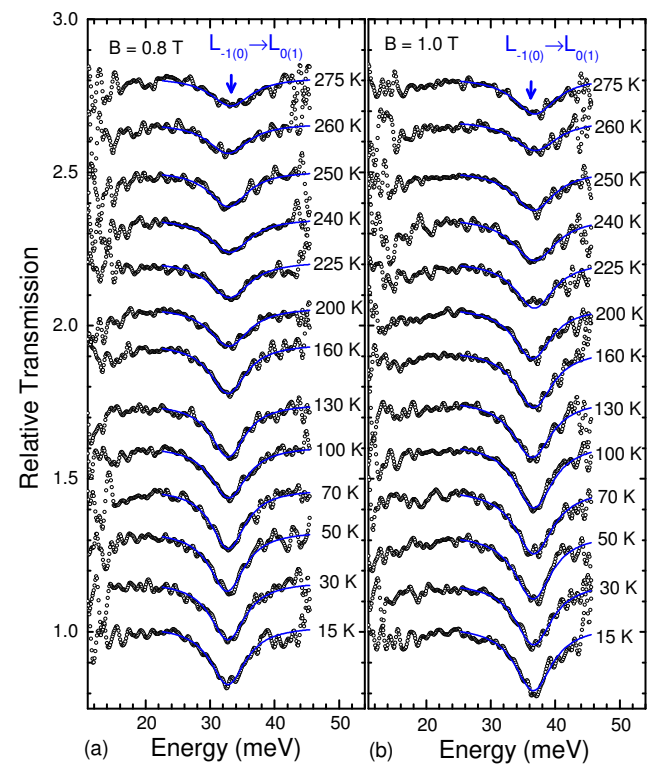

FIG. 2: (color online) Temperature dependence of the transmission spectra taken at magnetic fields $B=0.8$ and $1.0 \mathrm{~T}$ in parts (a) and (b), respectively, showing the $\mathrm{L}_{-1(0)} \rightarrow \mathrm{L}_{0(1)}$ transition up to the room temperature. The blue curves represent the Lorentzian fits whose parameters are plotted in Fig. 3. For clarity, successive spectra in both parts are shifted vertically by 0.15 .

around $10^{12} \mathrm{~cm}^{-2}$ but this time decreases rapidly with decreasing carrier density 14. The scattering time of $\tau \sim 260 \mathrm{fs}$ was also reported in charged graphene layer $\left(4 \times 10^{12} \mathrm{~cm}^{-2}\right)$ at $\mathrm{SiC} /$ graphene interface [29]. Recently, scattering times $\tau \sim 100$ fs were achieved at density down to $\sim 10^{10} \mathrm{~cm}^{-2}$ in suspended graphene [11, 12].

The lowest field of $B=40 \mathrm{mT}$ for which the welldefined absorption line is observed at $\hbar \omega_{c} \approx 7 \mathrm{meV}$ allows an independent estimation of the lower bound for $\tau$, and the mobility $\mu$. Using the semi-classical condition $\omega_{c} \tau>1$, allowing the carriers to complete one cyclotron orbit without scattering, gives a minimum relaxation time $\tau>\hbar / 7 \mathrm{meV} \simeq 100 \mathrm{fs}$ independent of but in a good agreement with the previous estimation based on the linewidth $\delta E$. The condition $\omega_{c} \tau>1$ can be rewritten as $\mu B>1$, which leads to the mobility $\mu>0.25 \times 10^{6} \mathrm{~cm}^{2} /$ (V.s). Note that this value represents only the lowest bound, as the main absorption line disappears from the spectrum due to the complete filling (depopulation) of $n=1(n=-1)$ LL and not due to the LL broadening. It remains an open question how this mobility would evolve with increasing carrier density, since the scattering mechanisms are different from those in exfoliated graphene, where ionized impurities dominate and the mobility remains nearly constant [11, 12, 14, 17].

To estimate the conductivity, we can use the standard relation $\sigma(\varepsilon)=\left(e^{2} / 2\right) D(\varepsilon) \tilde{c}^{2} \tau$, derived in the Boltzmann transport theory. This formula is applicable for graphene systems with a homogeneous density of carriers [8, 9$]$,

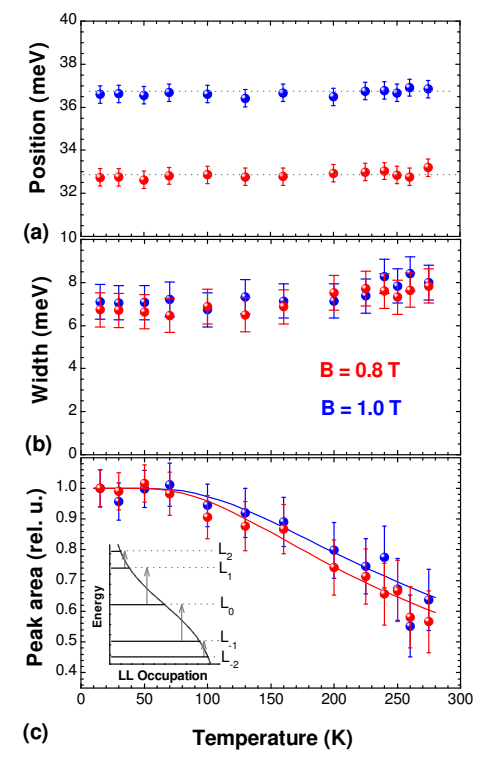

FIG. 3: (color online) Parameters of the Lorentzian fits to the transmission spectra in Fig. 2 Red and blue color represent data for $B=0.8$ and $1.0 \mathrm{~T}$, respectively. Whereas position and width of absorption lines in parts (a) and (b) show none or a very a weak temperature dependence, the peak area in the part (c) significantly decreases with $\mathrm{T}$, nearly following the theoretical expectation $1-2 f_{1}$ depicted by solid lines. The inset schematically shows the LL occupation and possible dipole-allowed absorption processes between LLs at finite temperature in undoped graphene $\left(E_{F}=0\right)$.

fulfilled in our case, but distinctive deviations from a presumably more precise self-consistent Born approximation are predicted in the immediate vicinity of Dirac point [13]. To calculate the density of states $D(\varepsilon)$, we recall that no deviation from the $\sqrt{B}$ scaling of the position of the main line is observed, and thus the expression $D(\varepsilon)=g_{s} g_{v}|\varepsilon| /\left(2 \pi \tilde{c}^{2} \hbar^{2}\right)$ remains valid down to a few meV from Dirac point, where $g_{s}=g_{v}=2$ denote the spin and valley degeneracy, respectively. Finally, we obtain the conductivity in the form $\sigma(\varepsilon)=\left(2 e^{2} / h\right)|\varepsilon|(\tau / \hbar)$. In the limit of a very low magnetic field, when the LL quantization energy is comparable to the LL broadening, we find $\delta E=2 \hbar / \tau \rightarrow \approx 3 \mathrm{meV}$ and the Fermi level $|\varepsilon| \rightarrow$ $\tilde{c} \hbar \sqrt{\pi n_{0}} \approx 8 \mathrm{meV}$ which implies a zero-field conductivity $\sigma=\left(4 e^{2} / h\right)(|\varepsilon| / \delta E) \approx 10 e^{2} / h$, slightly larger than the minimum conductivity reported in Refs. [2, 3, 14], but corresponding to conclusions for relatively clean samples [15, 17].

Having discussed the spectra taken at $T=2.0 \mathrm{~K}$, we turn our attention to measurements at elevated temperatures in Fig. 2. In these data, we can follow the evolution of the $\mathrm{L}_{-1(0)} \rightarrow \mathrm{L}_{0(1)}$ transitions at $B=0.8$ and $1.0 \mathrm{~T}$ in the interval of temperatures $T=15-275 \mathrm{~K}$. Apart from an apparent decrease in the absorption line intensity, no other effects are noticeable. Hence, the welldefined LLs are uniquely observed in graphene at room 
temperature and for magnetic fields below $1 \mathrm{~T}$, much lower than reported up to now [32]. The blue curves in Fig. 2 are Lorentzian fits to the data, and the obtained parameters, the peak position, width and area are plotted in Fig. 3. Based on these results, we can draw the following conclusions. The constant position of the absorption line shows that the Fermi velocity is independent of temperature. The extremely weak increase of the linewidth shows that no important scattering mechanism is thermally-activated, indicating that the mobility $\mu>0.25 \times 10^{6} \mathrm{~cm}^{2} /($ V.s) remains practically unchanged up to room temperature. For comparison, the mobility in standard 2D GaAs systems at room temperature does not exceed $10^{4} \mathrm{~cm}^{2} /(\mathrm{V} . \mathrm{s})$, being intrinsically limited by electron scattering on optical phonons 33]. Scattering on neutral centers, independent of carrier momentum and in consequence raising the temperature independent mobility, is likely the predominant scattering mechanisms in the investigated graphene layers which are well separated from the substrate and screened by highly conducting interface graphene sheets. Such mechanism is consistent with the above discussed observation of the nearly $\sqrt{B}$ dependence of the linewidth. For comparison, the scattering on ionized impurities determines carrier mobility in exfoliated graphene, which in contrast may lead to a temperature dependent mobility at high temperature, in case of a non degenerate gas.

The decrease in the strength of the transition can be understood by considering the influence of the thermal population and depopulation of LL's on the probability of the absorption process. The probabilities for the transitions $\mathrm{L}_{0} \rightarrow \mathrm{L}_{1}$ and $\mathrm{L}_{-1} \rightarrow \mathrm{L}_{0}$ are expressed as $P_{0 \rightarrow 1} \propto\left(f_{0}-f_{1}\right)$ and $P_{-1 \rightarrow 0} \propto\left(f_{-1}-f_{0}\right)$, respectively, yielding the total probability $P \propto\left(f_{-1}-f_{1}\right)$, where $f_{n}$ is the occupation of the $n$-th LL. Assuming undoped graphene $\left(E_{F}=0\right)$, the relative decrease in the intensity of the main absorption line can be written as $I(T)=1-2 f_{1}$ which reproduces the data in Fig. 3(c) extremely well. To calculate $f_{1}$, the optically determined Fermi velocity $\tilde{c}=1.02 \times 10^{6} \mathrm{~m} . \mathrm{s}^{-1}$ was used. Hence, the only pronounced effect observed in our FIR spectra with increasing temperature is a population effect due to the thermal excitation of carriers, as schematically shown in the inset of Fig. 3(c).

In summary, MEG has been investigated using LL spectroscopy in low magnetic fields and at temperatures ranging from 2 to $275 \mathrm{~K}$. Well-defined inter-LL absorption are observed at low temperatures down to magnetic fields of $40 \mathrm{mT}$. The LL spectroscopy thus probes the electronic states in the immediate vicinity of Dirac point, which are hardly accessible in experiments on exfoliated graphene [11, 12, 14, 17] due to the inevitable presence of electron-hole puddles. The conductivity of nearly neutral MEG deduced from our optical data is found to be consistent with recent theory [9, 15]. The temperature independent width of the spectral lines, which arise from the temperature independent LL broadening, indicate very weak thermally activated scattering processes. Therefore, the carrier mobility in excess of $250,000 \mathrm{~cm}^{2} /(\mathrm{V} . \mathrm{s})$, evaluated from low temperature data, remains practically constant with increasing temperature, confirming the recent expectations of record room-temperature mobilities in graphene 23]. MEG is an interesting material to probe the properties of Dirac particles in the immediate vicinity of Dirac point and has a potential as a platform for high performance graphene-based electronics. However, realization of any electric transport experiments on nearly neutral MEG will require passivation of the highly conducting graphene interface layers. Encouraging research along these lines is already underway [34].

The present work was supported by Contract No. ANR- 06-NANO-019, Projects No. MSM0021620834 and No. KAN400100652, and by the European Commission through Grant No. RITA-CT-2003-505474. Support is acknowledged from NSF-NIRT 4106A68 and NSF-MRI 4106A95 grants and from the W. M. Keck foundation. A USA-France travel grant from CNRS is acknowledged.

* Electronic address: orlita@karlov.mff.cuni.cz

[1] C. Berger et al., J. Phys. Chem. B 108, 19912 (2004).

[2] K. S. Novoselov et al., Nature 438, 197 (2005).

[3] Y. B. Zhang, Y. W. Tan, H. L. Stormer, and P. Kim, Nature 438, 201 (2005).

[4] C. Berger et al., Science 312, 1191 (2006).

[5] A. K. Geim and K. S. Novoselov, Nature Mater. 6, 183 (2007).

[6] A. H. Castro Neto, F. Guinea, N. M. R. Peres, K. S. Novoselov, and A. K. Geim, Rev. Mod. Phys. p. to be published (2008).

[7] T. Ando, J. Phys. Soc. Jap. 75, 074716 (2006).

[8] K. Nomura and A. H. MacDonald, Phys. Rev. Lett. 98, 076602 (2007).

[9] E. H. Hwang, S. Adam, and S. DasSarma, Phys. Rev. Lett. 98, 186806 (2007).

[10] J. Martin et al., Nature Phys. 4, 144 (2008).

[11] K. I. Bolotin et al., Solid State Commun. 146, 351 (2008).

[12] X. Du, I. Skachko, A. Barker, and E. Y. Andrei, Nature Nanotech. 3, 491 (2008).

[13] N. H. Shon and T. Ando, J. Phys. Soc. Jap. 67, 2421 (1998).

[14] Y.-W. Tan et al., Phys. Rev. Lett. 99, 246803 (2007).

[15] S. Adam, E. H. Hwang, V. M. Galitski, and S. D. DasSarma, Proc. Natl. Acad. Sci. USA 104, 18392 (2007).

[16] V. V. Cheianov, V. I. Fal'ko, B. L. Altshuler, and I. L. Aleiner, Phys. Rev. Lett. 99, 176801 (2007).

[17] J.-H. Chen et al., Nature Phys. 4, 377 (2008).

[18] J. Hass et al., Phys. Rev. B 75, 214109 (2007).

[19] J. Hass et al., Phys. Rev. Lett. 100, 125504 (2008).

[20] F. Varchon et al., Phys. Rev. Lett. 99, 126805 (2007).

[21] M. L. Sadowski, G. Martinez, M. Potemski, C. Berger, and W. A. de Heer, Phys. Rev. Lett. 97, 266405 (2006).

[22] S. Frank, P. Poncharal, Z. L. Wang, and W. A. de Heer, Science 280, 1744 (1998). 
[23] S. V. Morozov et al., Phys. Rev. Lett. 100, 016602 (2008).

[24] C. Faugeras et al., Appl. Phys. Lett. 92, 011914 (2008).

[25] A. C. Ferrari et al., Phys. Rev. Lett. 97, 187401 (2006).

[26] Z. Jiang et al., Phys. Rev. Lett. 98, 197403 (2007).

[27] Z. Li et al., Nature Physics 4, 532 (2008).

[28] M. Orlita et al., Phys. Rev. Lett. 100, 136403 (2008).

[29] X. Wu, X. Li, Z. Song, C. Berger, and W. A. de Heer, Phys. Rev. Lett. 98, 136801 (2007).
[30] P. Plochocka et al., Phys. Rev. Lett. 100, 087401 (2008).

[31] E. A. Henriksen et al., Phys. Rev. Lett. 100, 087403 (2008).

[32] K. S. Novoselov et al., Science 315, 1379 (2007).

[33] W. Walukiewicz, H. E. Ruda, J. Lagowski, and H. C. Gatos, Phys. Rev. B 30, 4571 (1984).

[34] X. Wu et al., Phys. Rev. Lett. 101, 026801 (2008). 